\title{
IMPACT OF MACROCELLULAR NETWORK DENSIFICATION ON THE CAPACITY, ENERGY AND COST EFFICIENCY IN DENSE URBAN ENVIRONMENT
}

\author{
S. F. Yunas, T. Isotalo, J. Niemelä and M. Valkama \\ Department of Electronics and Communications Engineering, \\ Tampere University of Technology, Tampere, Finland
}

\begin{abstract}
This paper aims to show the effect of macrocellular network densification on the capacity, energy and cost efficiency. The presented results are based on radio propagation simulations that consider macrocellular network with different inter-site distances, i.e. different site densities, and also take into account the presence of indoor receiver points by varying outdoor and indoor receiver distribution. It is observed that as a result of densifying the network, the cell spectral efficiency reduces due to increasing level of inter-cell interference. However, as a result of densification, the network area capacity can be improved since the area spectral efficiency increases. Nevertheless, the densification efficiency decreases because of the reduction of cell spectral efficiency, especially when indoor receiver points are taken into account. The results hence indicate that densification of macrocellular network suffers from inefficiency which results in higher energy and cost per bit per Hertz, and thus calls for alternative methods to deploy networks, or alternatively, more sophisticated methods, such as base station coordination or inter-cell interference cancellation techniques, to be implemented for future cellular networks.
\end{abstract}

\section{KEYWORDS}

Macrocell densification, area spectral efficiency, energy efficiency, cost efficiency, 3D ray tracing model

\section{INTRODUCTION}

Macrocellular networks have been and still continues to be the basis for cellular network deployments globally. High power transmitters with highly elevated and directive antenna array positions are superior in terms of wide-area coverage provisioning. They also play a major role in fulfilling the mobility demands of cellular users, and hence, are assumed to maintain their position in the future as well. Moreover, it is envisioned that macrocell networks will continue to provide the outdoor coverage layer with small cells satisfying the local outdoor and indoor capacity demands. Current cellular networks are inherently heterogeneous in terms of network configuration and this trend is building towards an even denser heterogeneous configuration as new small cell technology and different other indoor network solutions become more and more common.

The global cellular traffic has been on a steady rise since the early days of $2 \mathrm{G}$ networks. With the increasing popularity of mobile broadband subscriptions, the projected growth in the capacity demand has been estimated to increase 15 folds in the upcoming five years [1]. Fundamentally, the main mechanisms to increase the network capacity are increased link and radio resource management efficiency together with utilization of wider bandwidth and the cell size, i.e., having

DOI : 10.5121/ijwmn.2013.5507 
dense network configuration with small cells. The idea of enhancing the system capacity through network densification can be dated back to late 1940s when the cellular concept was introduced [2]. The initial adoption of the concept, however, was slow at first but started to gain serious attention when $2 \mathrm{G}$ networks were introduced. Since then, network densification has been viewed as a feasible pathway towards network evolution.

Lately, engineers and academic researchers have been studying and evaluating the concept with different performance enhancing techniques. In [3], the performance of macrocellular densification with different transmission schemes has been compared with a network employing base station coordination algorithms. The study concentrates on various techniques that can maximize the minimum spectral efficiency of the served users. In addition, a constant user density, irrespective of the network size, has been assumed. This results in a partially loaded system where some of the base stations are kept in sleep mode to avoid over provisioning of the network capacity. The results show that the cell spectral efficiency increases as the network is densified to a certain point and then saturates. In [4], the average cell spectral efficiency is shown to increase linearly with network densification in partially loaded system. The impact of macrocell densification on the network throughput and power consumption in both homogeneous and heterogeneous network environments has been studied in [5]. The study considers a fully loaded network, where all the base stations are continuously transmitting at full power. However, the maximum transmit power per base station is varied as the network is densified. The findings in [5] follow the outcomes of [4] i.e., in a homogeneous macrocell network, the cell spectral efficiency tends to improve with increasing network density. In [6, 7], the performance of homogeneous macrocellular network densification has been examined and compared with different heterogeneous network deployment alternatives. The papers take a slightly different approach by introducing variable traffic. Hence the system performance is evaluated in terms of served area traffic during busy hour. Unlike in [3-5], where only an outdoor environment is assumed, the studies in $[6,7]$, also take into account the indoor environment with buildings and users distributed among different floors. Nevertheless, their results indicate increasing served area traffic per busy hour as the network is densified.

While significant amount of time and effort in the last two decades was dedicated by the industry and academia in improving the spectral efficiency of wireless networks, more recently, the focal point of the industry has started to expand towards including energy and cost efficiency aspects into its domain. To cope with the current rate of 'exponentially' increasing capacity demand, deployment of several magnitudes more base stations will be required, which is considered by the industry to be a feasible pathway. However, this strategy is known to significantly increase the cost and energy consumption of the cellular networks. The ICT industry currently contributes around $2 \%$ of the global greenhouse gas emission and this contribution is expected to increase to $4 \%$ by the year 2020 [8]. According to some studies conducted in 2007/2008, the radio access networks alone had a share of around $0.3 \%-0.5 \%$ in the global CO2 emissions $[9,10]$ and out of this roughly $80 \%$ came from the base stations [11]. As the worldwide awareness regarding global warming increases, political initiatives at the international level have started to put requirements on the operators and manufacturers to lower the gas emissions of communications networks [12]. This has led the telecommunication industry, especially the standardization and regulatory bodies, to focus their attention towards building 'greener' wireless networks. In the research community, considerable number of studies have been conducted and published in the recent years focusing on quantifying the energy consumption of the wireless networks by establishing different metrics for evaluation of the energy efficiency, proposing power consumption models for different base station types and ways to improving the power consumption of the networks while maintaining decent quality of service and system throughput. Studies emphasizing on the importance of having a holistic framework for evaluating the energy efficiency of the wireless networks have been reported in [13, 14]. In [13], the authors discuss the importance of evaluating the energy efficiency at each level of network hierarchy, namely component, link and network level, their 
mutual dependencies and the need for optimizing the system as a whole, rather than just focusing on improving only one aspect, for achieving an optimum system performance. Building up on the foundation laid in [13], the authors propose an energy efficiency evaluation framework in [14] for evaluating nationwide energy-efficiency of a mobile operator. The framework constitutes a power consumption model for different LTE base station types together with a proposed large scale deployment and long term traffic model. In [11], a new metric, area power consumption, is proposed to evaluate and compare the energy efficiencies of networks with different cell ranges (varying cell site densities $/ \mathrm{km}^{2}$ ). A brief overview of the energy efficiency metrics at different levels of a wireless network/system has been provided in [15]. The impact of cell size on the power consumption has been studied in [4] and [16-18] for different deployment strategies. However, the results in [16] differ with the other studies. The authors in [16] report that large cell deployments are efficient in terms of area power consumption as compared to small cells while the finding of [4], [17] and [18] claim otherwise. The contradictory results reported in the above studies come from differences in the power consumption models utilized in the studies and using different energy efficiency metrics that capture different statistics of the network [19]. The impact of network densification on the energy efficiency in the wireless networks has been investigated in [19]. Unlike the previous studies in [4] and [16-18], which fail to take into account the impact of interference and system throughput while evaluating the energy efficiency, the studies in [19] investigate the relation between energy efficiency, area capacity and cell size by taking into consideration both the interference and noise, and takes relates the energy efficiency in terms of system throughput. The paper refines the analytical power consumption model proposed in [11] to include the backhaul power consumption as well which has significant impact when considering dense deployments [20]. The studies reported in [15-19] have a common short come, i.e. they all use a simple analytical model that combines the power consumption of components into two or three parameters and hence do not accurately model the influence of each component, within the base station, on the total power consumption. In [14], the power consumption of core components that have significant impact on the total power consumption of the base station, have been separately modelled. Nevertheless, it still fails to take into account the backhaul power consumption in its model, which is a key in denser deployments, as mentioned in [20]. This problem has been addressed in [21], where the proposed analytical model takes into account the contribution of the core components within the base station as well as the influence of backhaul transmission unit. The performance of the proposed power consumption model is further validated by comparing it with measurements from a live $3 \mathrm{G}$ network.

In this paper, we study the downlink capacity performance of macrocellular network densification in a full load condition, which is the worst case scenario and also a typical methodology that is used for network capacity dimensioning. Unlike in [4] and [5], where the base station parameters (antenna height, transmit power) are varied with respect to the cell size, we keep the antenna height and transmit power unchanged; only the downtilt angle is varied as network is densified. Further, an accurate deterministic 3D ray tracing model has been used in our study. For energyefficiency analysis we use the power consumption model given in [21]. The main target of the paper is to evaluate impact of site densification on the average cell and area spectral efficiency for outdoor and indoor receiver point locations, and also to evaluate whether macrocellular densification is a feasible pathway, in terms of energy and cost efficiency, towards the evolution of future cellular networks. The study aims to answer the question, how much system capacity gain can we achieve through macrocellular network densification and whether the capacity gain is enough lower the energy per bit and cost per bit to make pure macrocellular densification energy and cost efficient solution? The rest of the paper is structured as follows. We introduce the system model in Section 2 with the analysis methodology. Section 3 presents the results and performance analysis for capacity efficiency. In Section 4, the power consumption model for the base stations is introduced and the energy efficiency results and analyzed. Next, the cost modelling methodology is discussed in Section 5 and the cost efficiency results are analyzed. Finally, based on the analysis, concluding remarks are given in Section 6. 


\section{SYSTEM MODELLING}

This section presents the system model that we have used in our macrocellular densification studies. It starts with the introduction of the simulation environment and cell layouts. This is followed by a brief description of the simulation tool and the propagation model that was used in evaluations. Next, different network and site configuration parameters as antenna model together with average inter-site distance (ISD) and corresponding tilt angles are described. Finally, a list of general simulation parameters is provided.

\subsection{Simulation environment and cell layout}

To imitate a dense urban environment, we created a fictive Manhattan type grid city model (Figure 1). A hexagonal layout was used as the basis of our macrocellular deployment strategy, although the actual network layout determined by the Manhattan grid that does not allow a pure hexagonal cell layout. Each building has dimensions of $110 \mathrm{~m} \times 110 \mathrm{~m}$, a height of $40 \mathrm{~m}$ and comprises of 8 floors. The streets were selected to be $30 \mathrm{~m}$ wide. For indoor floor plan, an open office layout was chosen. It renders a hall area with no rooms, i.e. no hard obstruction for signal propagation except for the ceiling, floors and exterior walls.

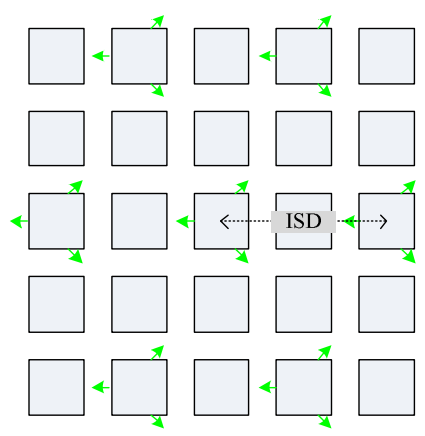

Figure 1. Manhattan grid city model (aerial view). The arrows show sector antenna positions and orientations (example case: ISD $297 \mathrm{~m}$ ). Note that only the first interfering tier is illustrated. The appropriate number of interfering tiers for each configuration used in the simulations is given in Table 2.

\subsection{Propagation model}

A commercial radio wave propagation tool (Wireless Insite), was used for the coverage prediction simulations. The outdoor and indoor radio channels are modelled using a 3D ray based model. The model employs a ray-launching technique based on 'Shooting and Bouncing Ray' (SBR) method to find the propagation paths through the 3D building geometry between a transmitter and receiver [22]. Rays are shot from the emitting source in discrete intervals and traced correspondingly as they reflect, diffract and transmit (penetrate) through and around the obstacles. Each ray is traced independently and the tracing continues until the maximum number of reflections is reached. Once all the propagation paths have been computed and stored, the field strength for each ray path is calculated using Uniform Theory of Diffraction (UTD) [23-25].

The accuracy of a 3D propagation model is dependent upon the input data and the total number of reflections, transmissions (or wall penetrations) and diffractions a single ray can encounter. To limit the calculation time, we used an empirical 'hit-and-trial' method, which involves simulating with a smaller number of interactions, and then re-simulating the same scenario by steadily increasing interactions and comparing the results. Once the results start to converge with 
insignificant change, those settings were then selected. In our case, this was observed at 10 reflections, 1 diffraction and 1 transmission where providing sufficiently accurate results.

To account for the outdoor-to-indoor propagation loss, the exterior wall direct penetration loss was chosen to be $25 \mathrm{~dB}$. The corresponding electrical properties of the exterior wall were found empirically by adjusting the conductivity $\sigma$, permittivity $\mathcal{E}$ and thickness of the wall, and observing the difference in the average signal level between several outdoor and indoor receiver points.

\subsection{Antenna models and positions}

An extended 3GPP antenna model based on [26] was adopted for simulations. The proposed version extends the original model of [13], which only considers the horizontal plane, and includes a vertical antenna pattern model with an option to set the electrical downtilt. The horizontal (azimuth) pattern, $G_{h}$, is given by:

$$
G_{h}(\varphi)=-\min \left[12\left(\frac{\varphi}{H P B W_{h}}\right)^{2}, F B R_{h}\right]+G_{m}
$$

where, $\varphi,-180^{\circ} \leq \varphi \leq 180^{\circ}$, is the azimuth angle relative to the main beam direction, $H P B W_{h}$ is the horizontal half power beam width $\left[{ }^{0}\right], F B R_{h}$ is the front-to-back ratio $[\mathrm{dB}]$ and $G_{m}$ is the maximum gain of the antenna $[\mathrm{dBi}]$. The vertical (elevation) pattern, $G_{v}$, is given by:

$$
G_{v}(\phi)=-\max \left[-12\left(\frac{\phi-\phi_{\text {etilt }}}{H P B W_{v}}\right)^{2}, S L L_{v}\right]
$$

where $\phi,-180^{\circ} \leq \phi \leq 180^{\circ}$, is the negative elevation angle relative to horizontal plane (i.e., $\phi=$ $-90^{\circ}$ is the upward plane relative to the main beam, $\phi=0^{0}$ is along the main beam direction, and $\phi=90^{\circ}$ is the downward plane relative to the main beam), $\phi_{\text {exilt }}$ is the electrical downtilt angle $\left[{ }^{0}\right]$, $H P B W_{v}$ is the vertical half power beam width $\left[{ }^{0}\right]$, and $S L L_{v}$ is the side lobe level $\left[{ }^{0}\right]$ relative to the maximum gain. The antenna parameter values were adopted from [26] except for electrical tilt angles which were based on the average inter-site distances.

The antennas were placed $2 \mathrm{~m}$ above the building roof, i.e. $42 \mathrm{~m}$ above the ground level. In order to ensure that the transmitted signal is not obstructed by the roof, sector antennas were placed either at corners of the buildings rather than at the center to ensure unobstructed propagation (see arrows in Figure 1).

\subsection{ISDs (cell density) and electrical tilt}

The cell density depends upon the average inter site distance (ISD or $\bar{d}_{\text {site }}$ ), which further specifies the dominance area of a cell. In our study, we define dominance area as the region where a cell provides highest signal level as compared to the rest of the cells. Altogether five different ISDs were considered. These were calculated from the center of the building (except in the average ISD of $170 \mathrm{~m}$ case, where it is calculated based on average inter cell distance owing to the square layout of the buildings).

Assuming a regular hexagon cell, the dominance area of a cell, $A_{\text {cell }}$ is given by:

$$
A_{\text {cell }}\left[k m^{2}\right]=\frac{\sqrt{3}}{6}\left(\bar{d}_{\text {site }}\right)^{2}
$$


The cell density, $\rho_{\text {cell }}$, per $\mathrm{km}^{2}$ is defined as $1 / A_{\text {cell }}$.

In order to avoid unnecessary interference into neighboring cells, the sectors are required to be down tilted. As mentioned earlier, the electrical tilt angle depends on the ISD as it defines the maximum cell range. Knowing the base station (BS) antenna height $\left(h_{B S}\right)$, the mobile station (MS) antenna height $\left(h_{M S}\right)$ and the cell range $\left(r_{\text {cell }}\right)$, the tilt angle was calculated geometrically as:

$$
\phi_{\text {etilt }}=\arctan \left(\frac{h_{B S}-h_{M S}}{r_{\text {cell }}}\right)
$$

The rest of the simulation parameters are gathered in Table 1. Note that the effective isotropic radiated power (EIRP) in the maximum antenna gain direction is $43 \mathrm{dBm}+18 \mathrm{dBm}=61 \mathrm{dBm}$. Moreover, for the receiver noise floor level calculation, a $20 \mathrm{MHz}$ bandwidth was assumed (nominal for long term evolution, LTE).

Table 1. General simulation parameters.

\begin{tabular}{lcc}
\hline Parameter & Unit & Value \\
\hline Operating frequency & {$[\mathrm{MHz}]$} & 2100 \\
Bandwidth, $W$ & {$[\mathrm{MHz}]$} & 20 \\
Transmit power at the antenna, $P_{T X}$ & {$[\mathrm{dBm}]$} & 43 \\
BS antenna beam width, $H P B W_{h / v}$ & {$[$ degrees $]$} & Directional $\left(65^{0} / 6^{0}\right)$ \\
MS antenna type & & Half-wave dipole \\
BS antenna gain & {$[\mathrm{dBi}]$} & 18 \\
MS antenna gain & {$[\mathrm{dBi}]$} & 2 \\
BS antenna height, $h_{B S}$ & {$[\mathrm{~m}]$} & 42 \\
MS antenna height, $h_{M S}$ & {$[\mathrm{~m}]$} & 2 \\
Receiver noise figure & {$[\mathrm{dB}]$} & 9 \\
Receiver noise floor, $P_{n}$ & {$[\mathrm{dBm}]$} & -92 \\
Propagation environment & & Manhattan \\
Propagation model & & $3 \mathrm{D}$ ray tracing \\
Building dimensions & {$[\mathrm{m}]$} & 110 x 110 \\
Building height & {$[\mathrm{m}]$} & 40 \\
Street width & {$[\mathrm{m}]$} & 30 \\
Indoor layout & & Open office \\
Outdoor-to-indoor wall penetration loss & {$[\mathrm{dB}]$} & 25 \\
\hline
\end{tabular}

\subsection{Analysis methodology}

Due to homogeneity of the environment, we only consider the receiver points from the dominance area of the center cell site for statistical analysis and then normalize the analysis to $1 \mathrm{~km}^{2}$ area. For simulating a continuous cellular network and analyzing its performance, it is necessary to take into account all the interfering cells that contribute to the interference level in the dominance area of a serving cell. In a realistic environment, the transmission path loss actually caps the number of receivable interfering sources at the serving cell which in turn limits the total interference level. However, in an ideal environment, like the Manhattan grid, the situation is exacerbated by the street canyons. The signals travelling in a street canyon tend to travel further as compared to the signal travelling in free space [27]. This is due to the tunneling effect caused by the side walls which direct the signals into the alley. As a consequence, the effect of distant 
interfering tiers especially the LOS (line of sight) tiers, which were negligible before, starts to become more visible.

To estimate the number of interfering tiers, only those tiers that had significant impact on the relative interference levels at the serving cell border (which is worst case scenario) are considered. This hit and trial method provides a fast and reliable estimate of the effective/dominant interfering tiers. Table 2 lists the average inter-site distance $\left(\bar{d}_{\text {site }}\right)$ and the corresponding electrical tilt angles, cell areas, cell densities (cells per $\mathrm{km}^{2}$ ), and the number of interfering tiers used in the simulations.

Table 2. ISD,electrical tilt, cell area, cell density and interfering tiers.

\begin{tabular}{ccccc}
\hline$\overline{\boldsymbol{d}}_{\text {site }}$ & $\phi_{\text {etilt }}$ & $\boldsymbol{A}_{\text {cell }}\left[\mathrm{km}^{2}\right]$ & $\boldsymbol{\rho}_{\text {cell }}$ per $\mathrm{km}^{2}$ & Interfering tiers \\
\hline $960 \mathrm{~m}$ & $3.5^{\circ}$ & 0.26 & 3.8 & 2 \\
$828 \mathrm{~m}$ & $4.1^{\mathrm{O}}$ & 0.2 & 5.1 & 2 \\
$593 \mathrm{~m}$ & $5.8^{\circ}$ & 0.1 & 9.9 & 3 \\
$297 \mathrm{~m}$ & $11.4^{\mathrm{O}}$ & 0.03 & 39.3 & 4 \\
$170 \mathrm{~m}$ & $47.5^{\circ}$ & 0.008 & 119.9 & 4 \\
\hline
\end{tabular}

\section{CAPACity EFFiCienCy ANALYSIS}

\subsection{SINR evaluation and mapping to Shannon capacity}

The performance of any cellular system or layout in a certain environment is highly dependent upon the radio propagation conditions. The quality of the radio link is determined by the coverage and the interference conditions which set a cap on the maximum throughput/users per cell, as defined by Shannon capacity bound, $C$ :

$$
C=W \log _{2}(1+\Gamma)
$$

where $W$ is the bandwidth of the system, $\Gamma$ is the signal-to-interference-noise ratio (SINR), which defines the radio propagation condition. From (5) it is evident that the cell/area spectral efficiency depends directly on $\Gamma$.

Assuming that there is no intra cell interference (typical assumption for frequency reuse 1 systems like orthogonal frequency division multiple access (OFDMA), where perfect orthogonality is assumed between the users of the same cell), the SINR at a $j^{\text {th }}$ receiver point (both outdoor and indoor) is calculated using the following relation:

$$
\Gamma_{j}=\frac{S_{j, \text { own }}}{\sum I_{j, \text { other }}+P_{n}}
$$

where $S_{j, \text { own }}$ is the received signal power from the own cell (serving cell) at $j^{\text {th }}$ receiver point, $I_{j, \text { other }}$ is the received interference power from the other cells at the $j^{\text {th }}$ receiver point, and $P_{n}$ is the noise floor level which includes the noise figure of the receiver as well.

In a multi-cellular scenario, a cell having the strongest signal level is considered as the serving cell and the rest are treated as interferers. For a set of $i$ cells reachable at the $j^{\text {th }}$ receiver, the best serving signal can be found as: 


$$
S_{j}=\underset{i}{\arg \max }\left(\operatorname{Pr}_{0 j}, \operatorname{Pr}_{1 j}, \ldots, \operatorname{Pr}_{i j}\right)
$$

where, $P r_{i j}$ is the received signal power from the $i^{\text {th }}$ center cell site at $j^{\text {th }}$ receiver.

\subsection{Cell spectral efficiency and area spectral efficiency}

We consider a fully loaded scenario in our simulation, i.e. all the base stations are transmitting at full power at all times. The cell spectral efficiency, $\eta_{\text {cell }}$, is defined as the maximum bit rate per Hertz that a cell can support under certain radio propagation conditions. For an area with a cell density of $\rho_{\text {cell }}$ and average cell spectral efficiency of $\bar{\eta}_{\text {cell }}$, the average area spectral efficiency, $\bar{\eta}_{\text {area }}$, for a fully loaded system with constant interferers is defined as [28]:

$$
\bar{\eta}_{\text {area }}\left[\text { bps } / \mathrm{Hz} \text { per } \mathrm{km}^{2}\right]=\rho_{\text {cell }} \times \bar{\eta}_{\text {cell }}
$$

where $\bar{\eta}_{\text {cell }}=\left\langle\frac{C}{W}\right\rangle$.

\subsection{Capacity efficiency result and analysis}

The general target of radio network planning is to design a network that provides sufficient coverage and maximizes overall capacity of the network with minimal costs. One of the most obvious methods for enhancing the network capacity is to increase the number of cells. However, the achievable average SINR ratios that eventually define the capacities (or 'cell spectral efficiency') in the cell level depend heavily on the network configuration. In this chapter, the results from the simulations, in terms of coverage, radio channel conditions and capacity, are analyzed and discussed.

Figure 2 provides the radio coverage statistics for the center cell site for both a) outdoor and b) indoor receiver points, for different ISDs. For the outdoor environment we can see that the signal levels improve in the whole cell area as macrocellular network is densified. Further densifying the network to ISD $170 \mathrm{~m}$ is shown to slightly degrade the coverage due to heavy downtilt angle $\left(47.5^{\circ}\right)$. For the indoor environment, the coverage pattern follows the same as in the outdoor. Again in the extreme densification scenario (ISD $170 \mathrm{~m}$ ), the coverage performance degrades even beyond ISD $969 \mathrm{~m}$.

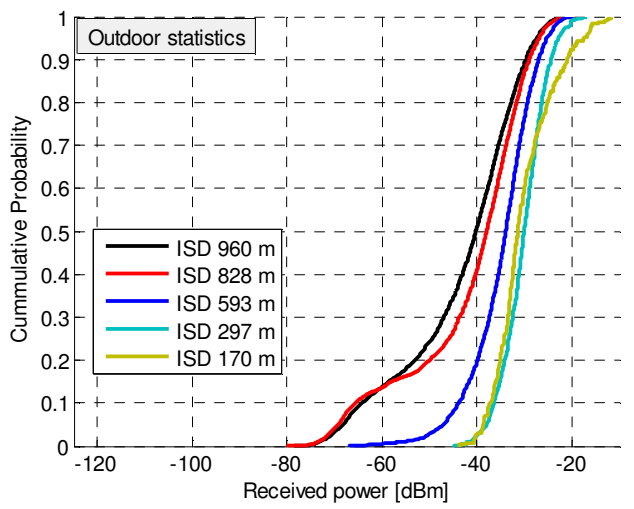

a)

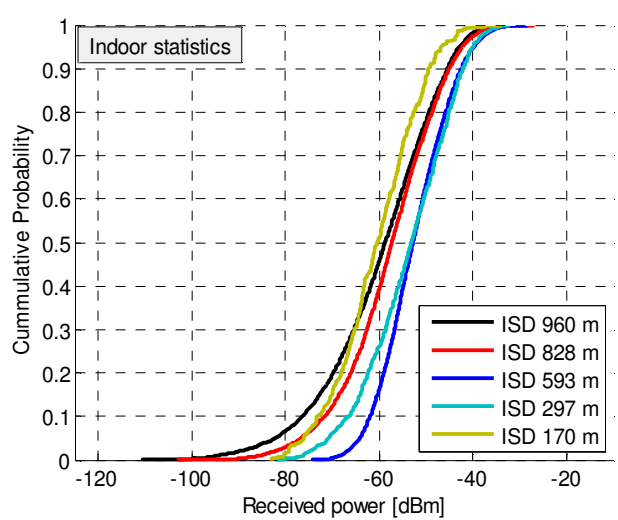

b)

Figure 2. CDF distribution of received signal strength for a) outdoor receiver pointsa and b) indoor receiver points. 
Figure 3 gives the center cell site statistics for the radio channel conditions (SINR) for both the outdoor (a) and indoor environment (b). In the outdoor environment, the radio channel conditions does not improve much in the overall cell, rather the SINR performance starts to degrade as we densify the network. The reduction in the SINR performance is more visible in the indoor environment with ISD $170 \mathrm{~m}$ having the worst interference conditions of all due to close proximity of the interfering cells.

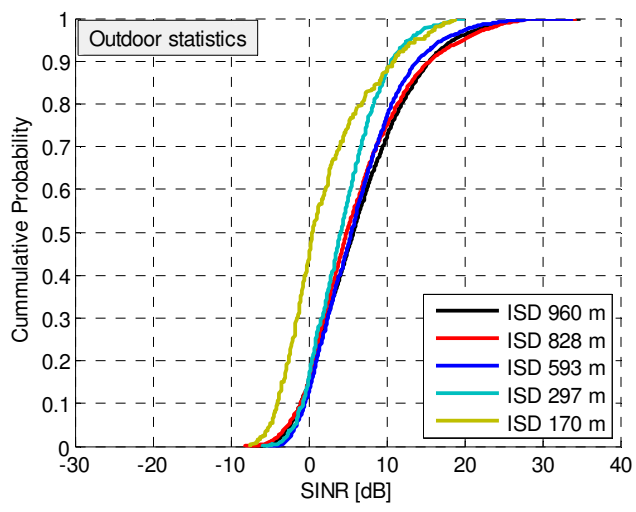

a)

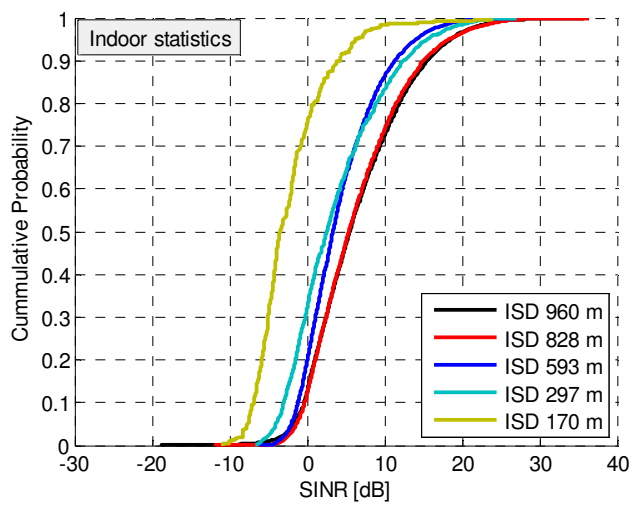

b)

Figure 3. CDF distribution for SINR [dB] for a) outdoor receiver points and b) indoor receiver points.

From the overall cell site capacity perspective, the improvement in the cell edge performance is of significance, as these regions, due to being away from the serving base station, experience worse radio propagation conditions. Hence, it is extremely important that the cell edge conditions in terms of cell capacities are as high as possible. The maximization of these edge conditions can be achieved with a proper radio network deployment that eventually minimizes the inter-cell interference caused by the overlap between adjacent or neighboring cells. Thus, in our analysis we will focus on the lower $10^{\text {th }}$ percentile values, which represent the conditions at the cell edge regions.

Figure 4 shows the statistical $10^{\text {th }}$ percentile values for the received signal levels (i.e., coverage) and SINR, respectively, for the outdoor (a) and different indoor floor levels (b). The x-axis indicates the cell density per $\mathrm{km}^{2}$ and $\mathrm{y}$-axis the corresponding received signal strength $[\mathrm{dBm}]$ or SINR [dB]. For analysis, we have grouped the indoor floor levels into three classes; the bottom floors, middle floors and the top floors. The bottom floors bar presents the average of the $10^{\text {th }}$ percentile values on the $1^{\text {st }}$ and the $2^{\text {nd }}$ floor, the middle floors bar indicate the average of the $10^{\text {th }}$ percentile values on the $4^{\text {th }}$ and the $5^{\text {th }}$ floor, while the top floors bar shows the average of the $10^{\text {th }}$ percentile values on $7^{\text {th }}$ and $8^{\text {th }}$ floor. From Figure $4 \mathrm{a}$ ) we can see that the outdoor receiver points experience quite high signal levels from the very beginning as compared to the indoor floors. The received signal levels are relative to receiver noise floor level which is at $-92 \mathrm{dBm}$ (as shown by the dashed line). For less densified configurations, the receiver points in the lower floors experience high signal losses as compared to ones on the top floors. However, as a result of densification of the network, the overall coverage levels start to improve. The improvement in the coverage level comes from the deployment of more base stations together with antenna down tilt that results in smaller cell sizes, thereby reducing the path losses. Subsequent densification of the network does not bring any further improvement in the indoor coverage, while the outdoor receiver points experience a moderate improvement in the average signal levels. In the extreme case of 120 cells $/ \mathrm{km}^{2}$ (or average ISD of $170 \mathrm{~m}$ ), the average signal levels saturate for receivers in outdoor and top floors, whilst the signal levels in the middle and lower floors start to experience 
coverage limitations. This is due to very high antenna tilt angles that cause signal losses in the lower floors.

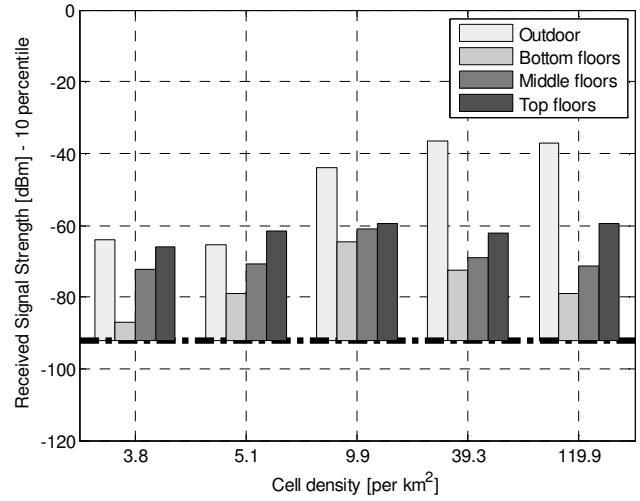

a)

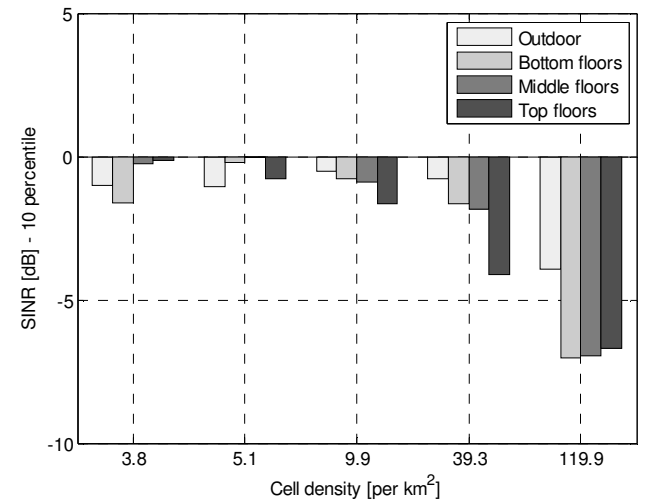

b)

Figure 4. Cell edge values (the $10^{\text {th }}$ percentile statistics) for a) received signal strength $[\mathrm{dBm}]$ and $\mathrm{b}$ ) SINR $[\mathrm{dB}]$.

Figure $4 \mathrm{~b}$ ) presents the $10^{\text {th }}$ percentile values i.e., the statistics from the cell edge, for SINR in outdoor and indoor environment for different cell densities. Although the coverage conditions on the top floor are better than in the middle and lower floors, the SINR performance degrades quite abruptly on top floor as the cell density increases. This is due to the rising interference conditions that become more prominent on the top floors as the network is densified. On the other hand, as a result of coverage improvement, the radio conditions in the lower and middle floor improve slightly when the network is densified to the level of 5 cells $/ \mathrm{km}^{2}$ (or average ISD of $828 \mathrm{~m}$ ). For more densified configurations, lower and middle floors start to become coverage and clearly interference limited.

Table 2. Capacity performance at the cell border region (the $10^{\text {th }}$ percentile values) for outdoor and different indoor floor levels.

\begin{tabular}{cccccc}
\hline \multicolumn{5}{c}{ Cell spectral efficiency, $\boldsymbol{\eta}_{\text {cell }},[\mathrm{bps} / \mathrm{Hz}]$} \\
\hline \multicolumn{5}{c}{ 10 percentile } \\
$\overline{\boldsymbol{d}}_{\text {site }}$ & $\boldsymbol{\rho}_{\text {cell }}$ per $\mathrm{km}^{2}$ & Outdoor & Bottom floors & Middle floors & Top floors \\
\hline $969 \mathrm{~m}$ & 3.8 & 0.84 & 0.76 & 0.96 & 0.98 \\
$828 \mathrm{~m}$ & 5.1 & 0.84 & 0.97 & 1 & 0.88 \\
$593 \mathrm{~m}$ & 9.9 & 0.92 & 0.88 & 0.86 & 0.75 \\
$297 \mathrm{~m}$ & 39.3 & 0.88 & 0.75 & 0.73 & 0.47 \\
$170 \mathrm{~m}$ & 119.9 & 0.49 & 0.26 & 0.27 & 0.28 \\
\hline
\end{tabular}

Table 4 provides the $10^{\text {th }}$ percentile values for the cell spectral efficiency versus cell densities, for the outdoor and different indoor floor levels. The SINR values under the dominance area of the center site are directly mapped to the cell spectral efficiency. In a full load condition, the cell efficiency is shown to decrease as the network is densified. Initially $\left(3.8\right.$ cells $\left./ \mathrm{km}^{2}\right)$, the cell edge spectral efficiency is at the level of $0.84 \mathrm{bps} / \mathrm{Hz}$ and reduces to the level of $0.49 \mathrm{bps} / \mathrm{Hz}$ for outdoor locations when network is densified to the level of $120 \mathrm{cells} / \mathrm{km}^{2}$. For the indoor floor levels, the overall cell edge efficiency is higher on the middle and top floors as compared to the lower floor levels and even outdoor location. However, as the network is densified to 120 cells $/ \mathrm{km}^{2}$, the cell spectral efficiency reduces to approximately $0.27 \mathrm{bps} / \mathrm{Hz}$ on all the floor levels. 
The higher degree of resource reuse due to denser deployments results in an increase of the area spectral efficiency as shown in Table 5. The impact of outdoor and indoor location on the area spectral efficiency is observed to be quite marginal in the beginning (ISD of $969 \mathrm{~m}$ and 828m), but as the network is densified, the difference in the area capacity gain starts to become more visible. For the cell spectral efficiency, the effect tends to get more recognizable when the network is densified beyond the level of 5 cells $/ \mathrm{km}^{2}$ (or average ISD of $828 \mathrm{~m}$ ). This is attributed to the rising indoor interference level, mostly on the top floors as shown in Figure 2b).

Table 3. Average cell and area spectral efficiency for different ISDs.

\begin{tabular}{|c|c|c|c|c|c|}
\hline \multirow[b]{2}{*}{$\overline{\boldsymbol{d}}_{\text {site }}$} & \multirow[b]{2}{*}{$\boldsymbol{\rho}_{\text {cell }}$ per $\mathrm{km}^{2}$} & \multicolumn{2}{|c|}{$\overline{\boldsymbol{\eta}}_{\text {cell }}[\mathrm{bps} / \mathrm{Hz}]$} & \multicolumn{2}{|c|}{$\overline{\boldsymbol{\eta}}_{\text {area }}\left[\mathrm{bps} / \mathrm{Hz} / \mathrm{km}^{2}\right]$} \\
\hline & & Outdoor & Indoor & Outdoor & Indoor \\
\hline $969 \mathrm{~m}$ & 3.8 & 2.7 & 2.67 & 15.1 & 14.96 \\
\hline $828 \mathrm{~m}$ & 5.1 & 2.65 & 2.61 & 22.42 & 22.06 \\
\hline $593 \mathrm{~m}$ & 9.9 & 2.57 & 2.05 & 36.05 & 28.74 \\
\hline $297 \mathrm{~m}$ & 39.3 & 2.09 & 1.99 & 92.81 & 88.06 \\
\hline $170 \mathrm{~m}$ & 119.9 & 1.65 & 0.88 & 289.2 & 153.9 \\
\hline
\end{tabular}

In mobile communications industry, it has been widely speculated that more than $70 \%$ of the overall network traffic originates from indoor users. Hence, to properly dimension its network a mobile operator has to consider service provisioning from the indoor perspective. However, the results indicate that macrocellular network densification in urban Manhattan environment clearly suffers from inefficiency indoors. If the radio network planning target is limited to coverage provisioning for outdoor users only, the densification efficiency is higher (see Figure 3a). On the other hand, if networks are planned for indoor coverage (as in practice), the efficiency is clearly lower. To illustrate this for a practical outdoor/indoor user distribution, we consider a scenario where majority of the receiver points are located indoors i.e., we assume the receiver distribution ratio as $20 \%$ outdoor and $80 \%$ indoor. To ensure statistically reliable results, the receiver points were randomly selected, with several iterations, from both outdoor and indoor environment with the intended ratio.

Figure 5 illustrates the capacity analysis in a slightly different way, where the relative area spectral efficiency for a network with different cell densities per $\mathrm{km}^{2}$ has been depicted. The area capacity values are relative with respect to nominal site density $\left(3.8 \mathrm{cells} / \mathrm{km}^{2}\right)$. The dashed line illustrates $100 \%$ densification efficiency $\left(\rho_{\text {eff }}\right)$ line, whereas the solid line shows the improvement of the area spectral efficiency for 20/80 \% outdoor/indoor receiver point distribution. For less dense configuration, there can be observed a linearly increasing trend in the area spectral efficiency. The densification efficiency is still roughly 0.8 for 9.9 cells $/ \mathrm{km}^{2}$ (or average ISD of $597 \mathrm{~m}$ ). However, beyond that point the efficiency can be observed to deteriorate significantly due to increase of inter-cell interference resulting in from network densification, and abruptly drops down to 0.38 for 119.9 cells $/ \mathrm{km}^{2}$ scenario. These results clearly illustrate the inefficiency of macrocellular network densification with a more practical user distribution. 


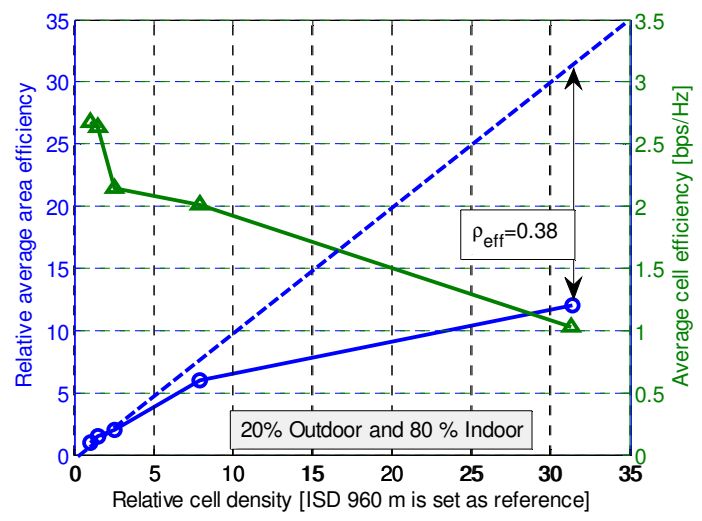

Figure 5. Relative area efficiency $(O)$ and average cell efficiency $(\triangle)$ vs. relative cell density (the dashed line indicate a linearly increasing area efficiency curve in an ideal case).

\section{ENERGY EFFICIENCY ANALYSIS}

\subsection{Power consumption modeling}

In order to estimate the energy efficiency of a macrocellular network over a certain area, it is important that the power consumption of a single base station (BS) site is modelled as accurately as possible. A base station site comprises of a base station unit, also known as the base transceiver station (BTS), which has the capability to transmit and receive radio signals to and from the mobile subscribers. It acts an interface between a subscriber and the core network, enabling the subscriber to wirelessly connect to the mobile operator's network. A macrocellular base station consists of various internal power consuming components that contribute to the overall energy usage. The components are power amplifier, transceiver, digital signal processing (DSP) chips and rectifier [14], [21]. Each sector of a base station has its own set of these components, hence the total power consumption scale with the number of sectors per base station. Apart from the internal components, there are some external equipment that also have significant contribution to the overall power usage of a macro base station cell site. The base stations require these external equipment for its smooth operation and connectivity to the mobile operator's core network, for instance, an air conditioning unit is required to maintain an ambient temperature (usually $25^{\circ} \mathrm{C}$ ) inside the base station shelter which houses the macro base station unit. Moreover, for connecting the base station to the backhaul network a transmission unit is installed. Note that the choice of transmission unit has considerable impact not only on the power consumption but also on the capital expenditure CAPEX (cost of equipment, deployment of backhaul network to the base station site etc.) and operating cost OPEX (power consumption, leased line rent etc.). For example, an optical transmission unit will consume less power and provides a very high capacity than a microwave transmission unit, but the associated cost of leasing or deploying an optical network increases the CAPEX considerably. In our study we consider a microwave link transmission unit, which is still widely used solution for mobile backhaul connectivity. For accurate estimation of the total network energy utilization, it is essential to also take into account the contributions from these external equipment.

An analytical power consumption model for a macrocellular base station site is proposed in [13] that takes into account the contribution from both internal base station components and external site equipment. The model further takes into account the impact of hourly network load on the total base station power utilization as well. As such the power consumption of a macro base station site, $P_{\text {consumption/Macro }}$, is given by [21]: 


$$
P_{\text {consumption/Macro }}[\text { Watt hour }]=P_{\text {constant }}+P_{\text {load }} \cdot F
$$

where $P_{\text {constant }}$ is the contribution from the internal components and external equipment whose power consumption is network load independent, $P_{\text {load }}$ is the contribution from the components/equipment whose power consumption depends on the instantaneous load of the network, and $F$ is the load factor varying from 0 to 1 , with 0 meaning 'no load' and 1 pertains to 'high load/full load'. The $F$ scales the power consumption of the load dependent base station components according to the network load per hour. In our analysis we use $F=1$, which is the worst case scenario, i.e. the network is running on a $100 \%$ load and all the load dependent components are consuming high power.

The load independent power consumption components include; rectifier $($ Rect $)$, microwave link (MLink) and air-conditioning (Air-cond) unit. Thus, $P_{\text {contant }}$ is given by:

$$
P_{\text {constant }}[\text { Watts }]=\left(n_{\text {sector }} \cdot P_{\text {Rect }}\right)+P_{\text {MLink }}+P_{\text {Air-Cond }}
$$

where $P_{\text {Rect }}$ is the power consumption of the rectifier, $P_{M L i n k}$ is the power consumption of a microwave link, and $P_{\text {Air-cond }}$ is the contribution from the air conditioning unit. As every sector of a macro base station has a separate rectifier, the total power consumption of the rectifier is scaled with the number of sectors, $n_{\text {sector }}$, installed at a base station.

The network load dependent power consumption components include power amplifier, transceiver and a baseband processing card (digital signal processing, DSP card). Thus, $P_{\text {load }}$ is given by:

$$
P_{\text {load }}[\text { Watts }]=n_{\text {sector }} \cdot\left[n_{\text {Ant }}\left(P_{\text {Amp }}+P_{T R X}\right)+P_{D S P}\right]
$$

where $P_{A m p}$ is the power consumption of the amplifier, $P_{T R X}$ is the power consumption of a transceiver and $P_{D S P}$ is the power usage contribution from the DSP card. The power contribution from these components is scaled with $n_{\text {sector }}$. Note that in case of MIMO (multiple input multiple output) configuration, a sector can have more than one antenna installed. Each antenna has a separate amplifier and a transceiver. Hence, the number of antennas per sector, $n_{A n t}$, also has an effect on the total power consumption. In our analysis we assume a SISO (single input and single output) configuration, i.e. 1 antenna per sector. The power consumption of the amplifier, $P_{\text {Amp }}$, depends on the input power requirement of the antenna, $P_{T X}$, and efficiency of the power amplifier, $\eta_{a m p}$ and is given by:

$$
P_{A m p}=\frac{P_{T X}}{\eta_{a m p}}
$$

Table 6 summarizes the input parameters for the base station power consumption model. The parameters are approximate values of the power consumption of different base station components and external equipment taken from [21], except for the power amplifier efficiency value which is typically $30 \%$ to $55 \%$ for the base stations. Using the input values in Table 3 , the power consumption of a 3 -sectored macro cellular base station operating in a full load condition is approximated to be 1338 Watts. 
Table 6. Input parameters for the power consumption model.

\begin{tabular}{lcc}
\hline Component/Equipment & Unit & Value \\
\hline Number of sectors, $n_{\text {sector }}$ & & 3 \\
Number of antennas per sector, $n_{A n t}$ & & 1 \\
Transmit power at the antennas, $P_{T X}$ & {$[$ Watts] } & 20 \\
Power consumption of DSP chip, $P_{D S P}$ & {$[$ Watts] } & 100 \\
Power Amplifier efficiency, $\eta_{A m p}$ & {$[\%]$} & 45 \\
Power consumption of Transceiver, $P_{T R X}$ & [Watts] & 100 \\
Power consumption of Rectifier, $P_{\text {Rect }}$ & [Watts] & 100 \\
Power consumption of Air-conditioning unit, $P_{\text {Air-cond }}$ & [Watts] & 225 \\
Power consumption of Microwave-Link unit, $P_{\text {MLink }}$ & [Watts] & 80 \\
\hline
\end{tabular}

\subsection{Area power consumption and energy-efficiency}

One of the most commonly used metric for assessing the energy efficiency of a network is by evaluating the energy-to-bit ratio performance of the network, i.e. the amount of energy consumed in transmitting one bit of information. On a network level, this relates to the total power consumed by the network in providing an aggregate network capacity. This methodology is appropriate for assessing the energy efficiency of a network operating under full load condition [13].

For studying the impact of base station site densification on the energy efficiency of the network, we first find the area power consumption by normalizing the total power consumption of a base station given in (9) to $1 \mathrm{~km}^{2}$ area. The normalized area power consumption of a macro cellular base station, $P_{\text {consumption } / \mathrm{km}^{2}}$, is given by:

$$
P_{\text {consumption } / \mathrm{km}^{2}}\left[\text { Watts } / \mathrm{km}^{2}\right]=\frac{P_{\text {consumption } / \text { Macro }}}{A_{\text {site }}}
$$

where $A_{\text {Site }}$ is the area of a base station (in $\mathrm{km}^{2}$ ) and is defined as $3 \times A_{\text {cell }}$ for a 3 sectored base station. The area power consumption is deemed as an appropriate metric in a case where the network is operating below its full load capacity, and the target is to minimize the power consumption over an area [13]. Hence, we use the energy-bit-ratio metric in our analysis. Finally, we define the energy-efficiency as the power consumed in transmitting one bps/ $\mathrm{Hz}$ and is calculated as following:

$$
E_{\text {eff }}[\text { bps } / \mathrm{Hz} / \mathrm{kW}]=\frac{\bar{\eta}_{\text {area }}}{P_{\text {consumption } / k m^{2}}}
$$

where $\bar{\eta}_{\text {area }}$ is the average area spectral efficiency as defined in (8).

\subsection{Energy efficiency results and analysis}

Table 7 summarizes the energy efficiency results for different inter site distances (varying cell densities) for outdoor and indoor receiver points. As we can note from the results, the power consumption per $\mathrm{km}^{2}$ increases with the increase in the cell density. This is because the area power consumption depends on the coverage area of the base station. We assume that the network coverage is continuous, without any coverage holes, and that the base stations consume same amount of power irrespective of the coverage area size. Hence, densification of the network leads 
to increased power consumption per area proportionally with the increase in number of base stations.

Table 7. Area power consumption and energy efficiency for different ISDs.

\begin{tabular}{ccccccc}
\hline \multirow{2}{*}{$\overline{\boldsymbol{d}}_{\text {site }}$} & \multirow{2}{*}{$\boldsymbol{\rho}_{\text {cell }}$ per $\mathrm{km}^{2}$} & $P_{\text {consumption } / \mathrm{km}^{2}}\left[\mathrm{~kW} / \mathrm{km}^{2}\right]$ & \multicolumn{2}{c}{$\overline{\boldsymbol{\eta}}_{\text {area }}\left[\mathrm{bps} / \mathrm{Hz} / \mathrm{km}^{2}\right]$} & \multicolumn{2}{c}{$\boldsymbol{E}_{\text {eff }}[\mathrm{bps} / \mathrm{Hz} / \mathrm{kW}]$} \\
\cline { 4 - 7 } & & 1.7 & Outdoor & Indoor & Outdoor & Indoor \\
\hline $969 \mathrm{~m}$ & 3.8 & 2.3 & 15.1 & 14.96 & 8.9 & 8.8 \\
$828 \mathrm{~m}$ & 5.1 & 4.4 & 22.42 & 22.06 & 9.7 & 9.6 \\
$593 \mathrm{~m}$ & 9.9 & 17.6 & 96.05 & 28.74 & 8.2 & 6.5 \\
$297 \mathrm{~m}$ & 39.3 & 53.5 & 289.2 & 88.06 & 5.3 & 5 \\
$170 \mathrm{~m}$ & 119.9 & & & & & \\
\hline
\end{tabular}

By densifying the network, the spectrum resource are reused more frequently, which thereby improves the area spectral efficiency. However, looking at the impact of site densification on the energy efficiency of the network, it is noted that although increasing the number of $\mathrm{bps} / \mathrm{Hz} / \mathrm{km}^{2}$, the energy needed to transmit $1 \mathrm{bps} / \mathrm{Hz}$ also increases as we densify our network, especially in the indoor environment. As an example, consider the initial case of 3.8 cells $/ \mathrm{km}^{2}$ (ISD $960 \mathrm{~m}$ ), where the average area spectral efficiency is the same for both outdoor and indoor environment. In this case, the total power consumed per $\mathrm{km}^{2}$ is approximately $1.7 \mathrm{~kW}$, which leads to energy efficiency of approximately $8.9 \mathrm{bps} / \mathrm{Hz} / \mathrm{kW}$ for outdoor and $8.8 \mathrm{bps} / \mathrm{Hz} / \mathrm{kW}$ for indoor environment. Upon decreasing the inter-site distance to $828 \mathrm{~m}$ (i.e., 5.1cells $/ \mathrm{km}^{2}$ ), a slight improvement can be observed in the energy efficiency $(9.7 \mathrm{bps} / \mathrm{Hz} / \mathrm{kW}$ for outdoor and 9.6 $\mathrm{bps} / \mathrm{Hz} / \mathrm{kW}$ for indoor). This improvement comes from the fact that in the initial stages of densification, the macrocellular network is slightly coverage limited. Hence, by densifying the network, the coverage levels improve in both outdoor and indoor environment, thereby improving the radio channel conditions and hence permitting higher cell spectral efficiency. Subsequent densification down to ISD $593 \mathrm{~m}$ and $297 \mathrm{~m}$ starts to degrade the energy efficiency performance as the network becomes more and more interference limited. The impact of degradation is more visible in the indoor environment due to relatively low rate of spectral efficiency improvement as compared to the outdoor environment. Eventually, when we densify the network to an extreme case (ISD $170 \mathrm{~m}$ case or 120 cells $/ \mathrm{km}^{2}$ ), given approximately 32 times more cells $/ \mathrm{km}^{2}$ as compared to initial ISD $969 \mathrm{~m}$ case, the area power consumption increases significantly. However at this stage, a slight improvement in the outdoor energy efficiency can be observed, but for the indoor environment the degradation in the energy efficiency performance extends even further. The reason is attributed to the capacity inefficiency in macrocellular networks.

As discussed in the previous section, the macrocellular network densification suffers from capacity in-efficiency in the indoor environment. The relative indoor capacity gain that we can achieve when we densify the network from $3.8 \mathrm{cell} / \mathrm{km}^{2}$ to approximately 120 cells $/ \mathrm{km}^{2}$ (32 times more cells) is only $38 \%$, which means that although the power consumption per $\mathrm{km}^{2}$ is increasing as we are increasing the number of cell density, the associated area capacity in the indoor environment to offset this increase of power consumption is not enough, hence the Watts/bit increases. Looking at the big picture, we conclude that pure macrocellular network densification suffer from both outdoor and indoor energy inefficiency.

\section{Cost EFFICIENCY AnALYSIS}

One of the most obvious ways to increase the capacity of a wireless network is by reusing the existing allocated spectrum as frequently as possible throughout the network service area, in other words by increasing the base station density. As such, the capacity of a cellular network is 
considered to be proportional to the base station density. Unfortunately, the network infrastructure cost also increases with the number of base stations which is a key concern for cost aware mobile operators that are striving to provide better services at lower cost in a highly competitive market. For any technological pathway to be feasible, the benefits must outweigh the incurred costs. Hence, correct estimation of the benefits and costs is very crucial. Cost efficiency analysis, or cost-benefit analysis, is one of the key methodologies that provide a general picture of the cost structure of an evolutionary pathway for a certain technology or system and whether or not it is a feasible option for investment. In this section we describe the cost modelling methodology used in our analysis, and based on the cost model we evaluate the cost-bit ratio for different ISDs (different site densities). Finally, we conclude the section by analyzing and discussing the cost-efficiency results.

\subsection{Cost modeling}

The cost of deploying a macrocellular network can be broadly divided into two types:

i. Investment cost or CAPEX (capital expenditure),

ii. Running/operational costs or OPEX (operational expenditure)

The CAPEX consists of equipment costs like radio base station, transmission equipment, antennas, cables, and site build out and installation cost. OPEX consists of site rental, electricity, transmission or leased line, and OA\&M (operation, administration \& maintenance). In addition to these, there can be cost components as such as radio network planning, core network and marketing costs whose impact can be modeled and taken into account as part of the radio network costs [29]. However, in the frame of this article, the scope is limited to items listed for CAPEX and OPEX as they typically depend very strongly on the number of deployed radio components. Combining CAPEX and OPEX gives the total cost of ownership (TCO) value of the deployed network.

The total cost structure of a mobile operator is dominated by the accumulated running costs i.e. the OPEX [30], which spans over the life-time of the network, while the CAPEX is considered during the initial network roll-out phase or when the network is upgraded. Thus, in order to account for both the CAPEX and OPEX in finding the 'total cost per base station' we use a standard economical method known as discounted cash flow (DCF) analysis, which gives the net present value (NPV) of the base station cost.

The net present value of the base station is simply found by summing up the discounted annual cash flow expenditure for a given study period (in years) [30, 31]. Mathematically;

$$
B S_{N P V}=\sum_{i=0}^{Y-1} \frac{c_{i}}{(1+r)^{i}}
$$

where $Y$ is the study period in years (typically 8 years for base stations value depreciation), $c_{i}$ is the total annual expenditure per base station (total annual cost which includes running cost and may include investment cost) in the $i^{\text {th }}$ year and $r$ is the discount rate which is assumed to be equal to $10 \%$. Table 8 gives the various cost items related to CAPEX and OPEX and their approximate values. The values have been adopted from [31-33]. 
To find the net present value of the base station we make the assumption that the mobile operator is deploying its network as a Greenfield project ${ }^{1}$ and that the whole network is deployed in the first year, so the CAPEX will be considered only for first year. Using the values in Table 8, the total cost per base station is $93 \mathrm{k€}$.

In order to assess the cost viability of the macrocellular network densification as an evolutionary pathway for future mobile broadband systems, we analyze the cost-bit ratio efficiency, or simply the cost-efficiency metric for the different ISDs. The cost efficiency is defined as the cost incurred in transmitting one bit/ $\mathrm{Hz}$ and is calculated as following:

$$
c_{\text {eff }}[\text { bps / Hz } / k €]=\frac{\bar{\eta}_{\text {area }}}{T_{\text {cost } / k m^{2}}}
$$

where $T_{\text {cost } / \mathrm{km}}{ }^{2}$ is the total area cost, i.e. the total cost of base stations over $1 \mathrm{~km}^{2}$ area and $\bar{\eta}_{\text {area }}$ is the average area spectral efficiency.

Table 8. CAPEX and OPEX related cost for a macro cellular base station.

\begin{tabular}{|c|c|}
\hline CAPEX (Initial costs) & \\
\hline Macro base station equipment & $10 \mathrm{k} €$ \\
\hline Site deployment cost & $5 \mathrm{k} €$ \\
\hline Total CAPEX & $15 \mathrm{kf}$ \\
\hline \multicolumn{2}{|l|}{ OPEX (Running costs) } \\
\hline Site rent (lease) & $5 \mathrm{k} € /$ year \\
\hline Electricity (power consumption charges) ${ }^{2}$ & $2.25 \mathrm{k} € /$ year \\
\hline Transmission line / Leased line rent ${ }^{3^{\circ}}$ & $0 \mathrm{k} € /$ year \\
\hline Operation, Administration \& Maintenance (OA\&M) & $5 \mathrm{k} € /$ year \\
\hline Total OPEX per annum & $12.25 \mathrm{kf}$ \\
\hline
\end{tabular}

\subsection{Cost efficiency results and analysis}

The cost efficiency analysis results for different macrocellular cell densities have been summarized in Table 9. As evident, the total cost of deployment per $\mathrm{km}^{2}$ increases as we increase the base station density. However, the important metric to investigate is not the aggregate cost but the cost per bit efficiency, i.e., we are interested in the relative gain that we can achieve from densification. In other words, we need to investigate whether the macrocellular densification can provide decent capacity gain to offset the incurred cost of deployment and hence bring down the cost per bit to make macrocellular densification a viable business case for investment.

In general, looking at the cost efficiency values in Table 9, it can be seen that it follows the energy efficiency performance pattern. In the initial stages (increasing the cell density from 3.8 to $5.1 \mathrm{cells} / \mathrm{km}^{2}$ ), there is a slight improvement in the cost efficiency performance for both outdoor and indoor environment. However, further densification not only degrades the cost efficiency performance but also the difference between outdoor and indoor environment starts to become more noticeable. This is, as mentioned previously, attributed to the inefficiency of pure macrocellular network densification in the indoor environment. From (15) it can be seen that the

\footnotetext{
${ }^{1}$ Any new network which is designed and deployed from scratch, i.e. operator has no prior deployment in that region. 2 The annual cost of electricity consumption is normally calculated based on total kilowatt hours (kWh) consumed during a given year. The cost per electricity unit is assumed to be $0.2 € / \mathrm{kWh}$. The calculated annual cost of electricity for macrocell base station given in Table 5 conforms quite well to the range given in [32].

${ }^{3} \mathrm{We}$ assume that the base stations are connected to the backhaul network via a microwave transmission link. Thus, there is no leased line (E1/T1) rent or deployment cost of fiber optic network.
} 
cost efficiency depends on the area spectral efficiency. The more the network is densified, the smaller capacity gain in the indoor environment is achieved, which results in higher cost per bit. For the outdoor environment, increasing the base station density to the extreme case (i.e., 120 cells $/ \mathrm{km}^{2}$ ) results in relatively higher area capacity gain than the indoor environment, which results in slightly improved cost efficiency. However, the efficiency still lags behind the cost efficiency of ISD $969 \mathrm{~m}$. Hence, it can be concluded that the pure macrocellular densification suffers from cost inefficiency especially in indoor environment in dense urban area.

Table 9. Total area cost and cost efficiency results for different ISDs.

\begin{tabular}{|c|c|c|c|c|c|c|}
\hline \multirow{2}{*}{$\overline{\boldsymbol{d}}_{\text {site }}$} & \multirow{2}{*}{$\boldsymbol{\rho}_{\text {cell }}$ per $\mathrm{km}^{2}$} & \multirow{2}{*}{$T_{\text {cost } / \mathrm{km}^{2}}\left[\mathrm{k} € / \mathrm{km}^{2}\right]$} & \multicolumn{2}{|c|}{$\overline{\boldsymbol{\eta}}_{\text {area }}\left[\mathrm{bps} / \mathrm{Hz} / \mathrm{km}^{2}\right]$} & \multicolumn{2}{|c|}{$\boldsymbol{c}_{e f f}[b p s / H z / k €]$} \\
\hline & & & Outdoor & Indoor & Outdoor & Indoor \\
\hline $969 \mathrm{~m}$ & 3.8 & 117 & 15.1 & 14.96 & 0.13 & 0.13 \\
\hline $828 \mathrm{~m}$ & 5.1 & 157 & 22.42 & 22.06 & 0.14 & 0.14 \\
\hline $593 \mathrm{~m}$ & 9.9 & 305 & 36.05 & 28.74 & 0.12 & 0.09 \\
\hline $297 \mathrm{~m}$ & 39.3 & 1224 & 92.81 & 88.06 & 0.08 & 0.07 \\
\hline $170 \mathrm{~m}$ & 119.9 & 3720 & 289.2 & 153.9 & 0.08 & 0.04 \\
\hline
\end{tabular}

\section{CONCLuSiON}

In this paper we have shown how macrocellular network densification reduces the cell spectral efficiency under full load conditions and varying receiver point distributions (outdoor/indoor) in dense urban environment using a 3D radio signal propagation model. As a result of reduction of cell spectral efficiency, the area spectral efficiency starts to saturate and macrocellular network densification becomes less efficient. However, if an operator targets only for outdoor coverage with macrocellular network, it still might be sufficient to fulfill the capacity demands through densification. From indoor coverage and capacity provisioning point of view, however, macrocellular network densification is clearly less efficient ( 0.38 densification efficiency with $20 / 80 \%$ outdoor/indoor receiver point distribution). The reduction in the spectrum efficiency also has a direct impact on the energy and cost efficiency of macrocellular network: lower spectrum efficiency in the indoor environment results in higher energy consumption and cost per bit. Moreover, from coverage point of view, in a dense urban environment with high rise buildings, the macrocellular network is not efficient in providing good coverage to indoor floors. This affects the attainable capacity in the indoor environment, and clearly further indicates that increasing network capacity demand will require alternative deployment strategies, as introduction of small cells (micro) or indoor (pico, femto), that will provide local indoor capacity within the network. On the macrocellular network, however, alternative mechanisms as interference mitigation techniques using smart antenna systems, base station transmission coordination or interference cancellation mechanisms are clearly needed to increase the densification efficiency.

Future work will concentrate on analyzing the coverage, capacity, costs and energy consumption of small or microcell networks and comparing that with macrocellular networks. Moreover, we will evaluate the effect of macrocellular network densification with base station coordination from capacity, costs, and energy-efficiency point of views. 
International Journal of Wireless \& Mobile Networks (IJWMN) Vol. 5, No. 5, October 2013

\section{ACKNOWLEDGEMENTS}

This research work has been financially supported by the Finnish Agency for Technology and Innovation (Tekes), under the Sino-Finland collaboration project "Energy-Efficient Wireless Networks and Connectivity Devices - Systems (EWINE-S)".

\section{REFERENCES}

[1] Traffic and Market Data Report, (2012), Annual report, Ericsson Inc.

[2] Macdonald, V. H., (1979), “Advanced Mobile Phone Service: The Cellular Concept”, The Bell System Technical Journal, Vol. 58.

[3] Liang, Y., et al., (2008), "Evolution of Base Stations in Cellular Networks- Denser Deployment versus Coordination", IEEE International Conference on Communications.

[4] Badic, B., Farrell T. O', Loskot, P., He J., (2009), "Energy Efficient Radio Access Architectures for Green Radio: Large versus Small Cell Size Deployment”, IEEE $70^{\text {th }}$ Vehicular Technology Conference (VTC).

[5] Richter, F., Fettweis, G., (2010), "Cellular Mobile Network Densification Utilizing Micro Base Stations", IEEE International Conference on Communications.

[6] Hiltunen, K., (2011), "Comparison of Different Network Densification Alternatives from the LTE Uplink Performance Point of View", IEEE $74^{\text {th }}$ Vehicular Technology Conference (VTC).

[7] Hiltunen, K., (2011), “Comparison of Different Network Densification Alternatives from the LTE Downlink Performance Point of View", IEEE International Symposium on Personal, Indoor and Mobile Radio Communications (PIMRC).

[8] ICT Sustainability Outlook: An Assessment of the Current State of Affairs and a Path Towards Improved Sustainability for Public Policies, White paper, (2013), BIO Intelligence services and Alcatel Lucent.

[9] Sustainable Energy Use in Mobile Communications, (2007), White paper, Ericsson Inc.

[10] Pickavet M., et al., (2008), "Worldwide Energy Needs for ICT: The Rise of Power-Aware Networking", International symposium on Advanced Networks and Telecommunications Systems.

[11] Richter F., et al., (2009), "Energy Efficiency Aspects of Base Station Deployment Strategies for Cellular Networks", IEEE $70^{\text {th }}$ Vehicular Technology Conference (VTC).

[12] EU Commissioner calls on the ICT industry to reduce its carbon footprint by $20 \%$ as early as 2015, MEMO/09/140. Press release, EU Commission (www.europa.eu)

[13] Correia, L.M., et al., (2010), "Challenges and Enabling Technologies for Energy Aware Mobile Radio Networks", IEEE Communications Magazine.

[14] Auer G., et al., "Cellular Energy Efficiency Evaluation Framework", IEEE $73^{\text {rd }}$ Vehicular Technology Conference (VTC).

[15] Chen T., et al., (2010), "Energy Efficiency Metrics for Green Wireless Communications", IEEE International conference on Wireless Communications and Signal Processing (WCSP).

[16] Ericson M., (2011), “Total Network Base Station Energy Cost vs. Deployment”, IEEE $73^{\text {rd }}$ Vehicular Technology Conference (VTC).

[17] Le T., and Nakhai M., (2010), "Possible power-saving gains by dividing a cell into tiers of smaller cells", IET Electroncis Letters, Vol. 46, No. 16.

[18] Leem H., Baek S. Y. and Sung D. K., (2010), “The Effects of Cell Size on Energy Saving, System Capacity, and Per-Energy Capacity”, IEEE Wireless Communications and Networking Conference. 
International Journal of Wireless \& Mobile Networks (IJWMN) Vol. 5, No. 5, October 2013

[19] Tombaz S., Sung K. W. and Zander J., (2012), "Impact of Densification on Energy Efficiency in Wireless Access Networks", IEEE Globecom Workshops.

[20] Tombaz S., et al., (2011), "Impact of backhauling power consumption on the deployment of heterogeneous mobile networks", IEEE Globecom conference.

[21] Deruyck M., Joseph W., and Martens L., (2012), "Power consumption model for macrocell and microcell base stations", Transactions on Emerging Telecommunications Technology.

[22] Schuster, J. and Luebbers, R., (1996), "Shooting and Bouncing Rays: Calculating the RCS of an arbitrarily shaped cavity", IEEE Transactions on Antenna and Propagation, Vol. 1.

[23] Keller, J. B., (1996), "Geometrical Theory of Diffraction”, Journal of Optical Society of America, Vol. 52, No. 2.

[24] Kouyoumjian, R. G., Pathak, P. H., (1974), “A Uniform Geometrical Theory of Diffraction for an edge in a perfectly conducting surface”, IEEE Proceedings, Vol. 62, No. 11.

[25] Balanis, C., (1989), Advanced Engineering Electromagnetics, John Wiley \& Sons Inc.

[26] Gunnarsson, F., et al., (2008), "Downtilted Base Station Antennas - A Simulation Model Proposal and Impact on HSPA and LTE Performance", IEEE $68^{\text {th }}$ Vehicular Technology Conference (VTC).

[27] Sridhara V., and Bohacek S., (2007), "Realistic propagation simulation of urban mesh networks", International Journal of Computer and Telecommunications network Computer Networks and ISDN Systems (COMNET).

[28] Alouni M., and Goldsmith A., (1999), "Area Spectral Efficiency of Cellular Mobile Radio Systems”, IEEE Transactions on Vehicular Technology, Vol. 48, No. 4, pp. 1047-1066

[29] Giles. T, et al., (2004), "Cost Drivers and Deployment Scenarios for Future Broadband Wireless Networks", IEEE $59^{\text {th }}$ Vehicular Technology Conference (VTC).

[30] Smura T., (2012), Techno-economic modelling of wireless network and industry architectures, Doctoral dissertation, Dept. Communications and Networking, Aalto University.

[31] Johansson K., et al., (2004), "Relation between base station characteristics and Cost structure in Cellular systems". IEEE International Symposium on Personal, Indoor and Mobile Radio Communications (PIMRC).

[32] Markendahl, J. and Ma $\square$ kitalo, O., (2010), “A comparative study of deployment options, capacity and cost structure for macrocellular and femtocells networks", IEEE International Symposium on Personal, Indoor and Mobile Radio Communications (PIMRC).

[33] Niemela J., and Isotalo T., (2012), Coverage constrained Techno-economical comparison of Macro and Small Cell Planning Strategies, Internal report, Dept. Electronics and Communications Engineering., Tampere University of Technology. 